\title{
Neues Hauptallergen der Hausstaubmilbe entdeckt
}

Die Diagnostik und spezifische Immuntherapie (SIT) einer Hausstaubmilbenallergie basiert auf natürlichen Allergenextrakten. Kommerziell verfügbare Extrakte weisen jedoch eine hohe Variabilität in der Allergenzusammensetzung und -menge auf, sodass es je nach individuellem Reaktivitätsprofil des Patienten zu falsch negativen Ergebnissen und einem mangelhaften Ansprechen auf die SIT kommen kann.

Einer Arbeitsgruppe um Dr. Susanne Vrtala vom Institut für Pathophysiologie und Allergieforschung der Medizinischen Universität Wien gelang es, ein neues Hauptallergen der Hausstaubmilbe dingfest zu ma- chen: Der $\mathrm{p} 23$ [Weghofer M et al. J Immunol 2013; 190: 3059-67]. Untersuchungen bei 347 Patienten mit Hausstaubmilbenallergie ergaben, dass Der p 23 bei $74 \%$ eine hohe IgE-Antikörper-Reaktivität aufweist, die mit den beiden bekannten Hauptallergenen Der $p 1$ und Der $\mathrm{p} 2$ vergleichbar ist. Darüber hinaus zeigte rekombinantes Der $\mathrm{p} 23$ eine hohe allergene Aktivität und erhöhte die Expression von CD203c auf Basophilen von Patienten mit Hausstaubmilbenallergie. Der $\mathrm{p} 23$ findet sich in Fäkalien der Milben, wird über die Luft übertragen und stellt so ein wichtiges respiratorisches Allergen dar. In kommerziellen Extrakten ist Der $\mathrm{p} 23$ je-

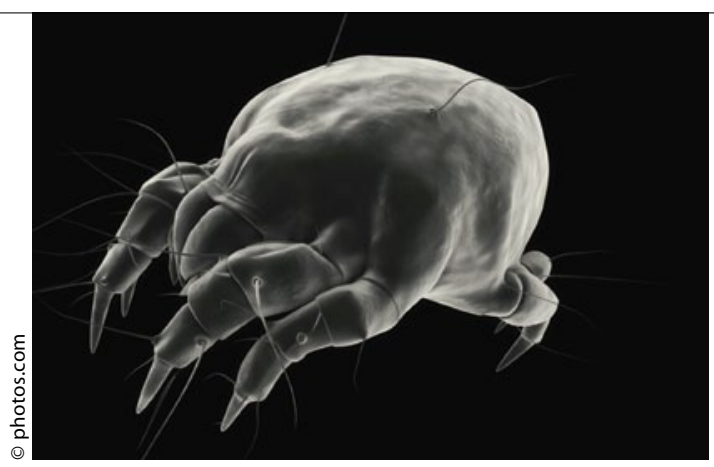

doch meist nicht enthalten, sagte Vrtala. Patienten, die vor allem auf Der $\mathrm{p} 23$ reagieren, können damit nicht detektiert und zufriedenstellend therapiert werden. Rekombinante Allergene könnten hier eine neue diagnostische und therapeutische Perspektive darstellen. Angelika Bauer-Delto

Vrtala S. Towards new forms of diagnosis and therapy of house dust mite allergy

\section{IgE-bindenden Epitopen von therapeutischen Antikörpern auf der Spur}

Immer häufiger werden Überempfindlichkeitsreaktionen auf verschiedene therapeutische Antikörper berichtet. Anaphylaktische Reaktionen auf Cetuximab werden durch präexistierende IgE-Antikörper gegen alphaGAL erklärt; eine Sensibilisierung könnte beispielsweise durch rotes Fleisch oder Zeckenstiche erfolgt sein.

Hinweise auf weitere allergene Epitope therapeutischer Antikörper fand nun eine Arbeitsgruppe um Prof. Dr. Uta Jappe vom Forschungszentrum Borstel. Deren Ziel ist es, gemeinsam mit Partnern aus Industrie und Universität Testsysteme zu entwickeln, die zur prädiagnostischen Erkennung von Unverträglichkeitsreaktionen auf Biologika eingesetzt werden können.

In einer Pilotstudie, vorgestellt von Dr. Sandra Minge, wurden die Seren von 19 Patienten, die nach Infusionen von Infliximab, Adalimumab, Etanercept sowie Cetuximab allergische Reaktionen gezeigt hatten, auf IgE-Reaktivität und -Spezifität hin analysiert. In vier von sieben Seren der Infliximab-Patienten konnten eindeutig AntiIgE-Infliximab-Antikörper nachgewiesen werden, bei zwei Patienten jedoch kein Anti-alpha-GAL-IgE. Spaltungsexperimente in Fab- und Fc-Fragmente ergaben, dass bei Cetuximab die lgE-bindenden Epitope auf der Fab-Region lokalisiert sind. Die Forscher vermuten, dass dies auch bei Infliximab der Fall ist und arbeiten derzeit an einem Epitopmapping, das weitere allergene Epitope identifizieren soll. Angelika Bauer-Delto

Minge S, Kromminga A, Platts-Mills T, Jappe U. Allergie auf Biologika: Pilotstudie zur Identifikation IgE-bindender Epitope

\section{Studie zeigt ernüchternde Versorgungsrealität bei Anaphylaxie}

Um Anaphylaxie-Patienten adäquat versorgen und anaphylaktische Reaktionen künftig verhindern zu können, ist eine zeitnahe Diagnostik erforderlich. Die Versorgungsrealität sei jedoch ernüchternd, berichtete Prof. Dr. Franziska Ruëff von der Klinik und Poliklinik für Dermatologie und Allergologie der Ludwig-Maximilians-Universität, München.

Eine retrospektive Auswertung der Daten von 710 Anaphylaxiepatienten der Allergieambulanz ergab, dass sich $42,6 \%$ erst nach zwei oder mehr anaphylaktischen Reaktionen vorstellten. Nur etwa die Hälfte suchte die Ambulanz innerhalb von 16 Wochen nach einer anaphylaktischen Episode auf, bei fast einem Viertel lag die letzte Reaktion aber schon mehr als ein Jahr zurück. Der Schweregrad der Reaktion spielte überraschenderweise für den zeitlichen Abstand zur Erstvorstellung keine Rolle, berichtete Ruëff. Bei einer Diagnostik innerhalb von 16 Wochen nach der anaphylaktischen Reaktion konnte in $68 \%$ der Fälle der Auslöser zweifelsfrei ermittelt werden; bei einer späteren Vorstellung sank diese Rate auf bis zu $48 \%$. Am häufigsten wurde eine Insektengiftallergie diagnostiziert. Obwohl bei fast allen diesen Patienten eine spezifische Immuntherapie indiziert war, kam nahezu jeder fünfte der Empfehlung nicht nach, darunter auch Patienten mit potenziell lebensbe- drohlichen Reaktionen in der Vorgeschichte. Die Krankheitswahrnehmung sei offensichtlich selbst bei schweren anaphylaktischen Reaktionen nicht besonders ausgeprägt, so Ruëff. Hier sei eine intensive Aufklärung über die Bedeutung einer zeitnahen Diagnostik und Therapie unerlässlich. Die aktuellen Ergebnisse sollten darüber hinaus Anlass für eine umfassende Versorgungsforschung geben.

Angelika Bauer-Delto

Ruëff F, Vollrath R, Reymus M, Walker A, Przybilla B. Versorgungsforschung bei Patienten mit Anamnese anaphylaktischer Reaktionen 